\title{
ON THE $\alpha$-SPECTRAL RADIUS OF UNIFORM HYPERGRAPHS
}

\author{
HaiYan GuO AND Bo Zhou ${ }^{1}$ \\ School of Mathematical Sciences \\ South China Normal University \\ Guangzhou 510631, P.R. China \\ e-mail: ghaiyan0705@163.com \\ zhoubo@scnu.edu.cn
}

\begin{abstract}
For $0 \leq \alpha<1$ and a uniform hypergraph $G$, the $\alpha$-spectral radius of $G$ is the largest $H$-eigenvalue of $\alpha \mathcal{D}(G)+(1-\alpha) \mathcal{A}(G)$, where $\mathcal{D}(G)$ and $\mathcal{A}(G)$ are the diagonal tensor of degrees and the adjacency tensor of $G$, respectively. We give upper bounds for the $\alpha$-spectral radius of a uniform hypergraph, propose some transformations that increase the $\alpha$-spectral radius, and determine the unique hypergraphs with maximum $\alpha$-spectral radius in some classes of uniform hypergraphs.
\end{abstract}

Keywords: $\alpha$-spectral radius, $\alpha$-Perron vector, adjacency tensor, uniform hypergraph, extremal hypergraph.

2010 Mathematics Subject Classification: 05C50, 05C65.

\section{REFERENCES}

[1] J. Cooper and A. Dutle, Spectra of uniform hypergraphs, Linear Algebra Appl. 436 (2012) 3268-3292.

doi:10.1016/j.laa.2011.11.018

[2] Y. Fan, Y. Tan, X. Peng and A. Liu, Maximizing spectral radii of uniform hypergraphs with few edges, Discuss. Math. Graph Theory 36 (2016) 845-856. doi:10.7151/dmgt.1906

[3] S. Friedland, S. Gaubert and L. Han, Perron-Frobenius theorem for nonnegative multilinear forms and extension, Linear Algebra Appl. 438 (2013) 738-749. doi:10.1016/j.laa.2011.02.042

\footnotetext{
${ }^{1}$ Corresponding author.
} 
[4] H. Guo and B. Zhou, On the spectral radius of uniform hypertrees, Linear Algebra Appl. 558 (2018) 236-249.

doi:10.1016/j.laa.2018.07.035

[5] H. Guo and B. Zhou, On the $\alpha$-spectral radius of graphs. ArXiv:1805.03456

[6] S. Hu, L. Qi and J. Xie, The largest Laplacian and signless Laplacian H-eigenvalues of a uniform hypergraph, Linear Algebra Appl. 469 (2015) 1-27. doi:10.1016/j.laa.2014.11.020

[7] C. Li, Z. Chen and Y. Li, A new eigenvalue inclusion set for tensors and its applications, Linear Algebra Appl. 481 (2015) 36-53. doi:10.1016/j.laa.2015.04.023

[8] H. Li, J. Shao and L. Qi, The extremal spectral radii of $k$-uniform supertrees, J Comb. Optim. 32 (2016) 741-764.

doi:10.1007/s10878-015-9896-4

[9] H. Li, J. Zhou and C. Bu, Principal eigenvectors and spectral radii of uniform hypergraphs, Linear Algebra Appl. 544 (2018) 273-285. doi:10.1016/j.laa.2018.01.017

[10] L.-H. Lim, Singular values and eigenvalues of tensors: a variational approach, in: Proceedings of the First IEEE International Workshop on Computational Advances of Multi-Sensor Adaptive Processing, Puerto Vallarta (2005) 129-132. doi:10.110SICAMAP.2005.1574201

[11] H. Lin, H. Guo and B. Zhou, On the $\alpha$-spectral radius of irregular uniform hypergraphs, Linear Multilinear Algebra, 2019. doi:10.1080/03081087.2018.1502253

[12] H. Lin, B. Mo, B. Zhou and W. Weng, Sharp bounds for ordinary and signless Laplacian spectral radii of uniform hypergraphs, Appl. Math. Comput. 285 (2016) $217-227$. doi:10.1016/j.amc.2016.03.016

[13] H. Lin, B. Zhou and B. Mo, Upper bounds for $H$ - and Z-spectral radii of uniform hypergraphs, Linear Algebra Appl. 510 (2016) 205-221. doi:10.1016/j.laa.2016.08.009

[14] V. Nikiforov, Merging the A- and Q-spectral theories, Appl. Anal. Discrete Math. 11 (2017) 81-107. doi:10.2298/AADM1701081N

[15] V. Nikiforov, G. Pastén, O. Rojo and R.L. Soto, On the $A_{\alpha}$-spectra of trees, Linear Algebra Appl. 520 (2017) 286-305. doi:10.1016/j.laa.2017.01.029

[16] C. Ouyang, L. Qi and X. Yuan, The first few unicyclic and bicyclic hypergraphs with largest spectral radii, Linear Algebra Appl. 527 (2017) 141-162. doi:10.1016/j.laa.2017.04.008 
[17] K.J. Pearson and T. Zhang, On spectral hypergraph theory of the adjacency tensor, Graphs Combin. 30 (2014) 1233-1248.

doi:10.1007/s00373-013-1340-x

[18] L. Qi, Eigenvalues of a real supersymmetric tensor, J. Symbolic Comput. 40 (2005) 1302-1324.

doi:10.1016/j.jsc.2005.05.007

[19] L. Qi, Symmetric nonnegative tensors and copositive tensors, Linear Algebra Appl. 439 (2013) 228-238.

doi:10.1016/j.laa.2013.03.015

[20] L. Qi, $H^{+}$-eigenvalues of Laplacian and signless Laplacian tensors, Commun. Math. Sci. 12 (2014) 1045-1064.

doi:10.4310/CMS.2014.v12.n6.a3

[21] L. Qi, J. Shao and Q. Wang, Regular uniform hypergraphs, s-cycles, s-paths and their largest Laplacian H-eigenvalues, Linear Algebra Appl. 443 (2014) 215-227. doi:10.1016/j.laa.2013.11.008

[22] J.Y. Shao, A general product of tensors with applications, Linear Algebra Appl. 439 (2013) 2350-2366. doi:10.1016/j.laa.2013.07.010

[23] S.K. Simić and B. Zhou, Indices of trees with a prescribed diameter, Appl. Anal. Discrete Math. 1 (2007) 446-454. doi:10.2298/AADM0702446S

[24] P. Xiao, L. Wang and Y. Lu, The maximum spectral radii of uniform supertrees with given degree sequences, Linear Algebra Appl. 523 (2017) 33-45. doi:10.1016/j.laa.2017.02.018

[25] P. Xiao, L. Wang and Y. Du, The first two largest spectral radii of uniform supertrees with given diameter, Linear Algebra Appl. 536 (2018) 103-119. doi:10.1016/j.laa.2017.09.009

[26] P. Xiao and L. Wang, The maximum spectral radius of uniform hypergraphs with given number of pendant edges, Linear Multilinear Algebra. doi:10.1080/03081087.2018.1453471

[27] Y. Yang and Q. Yang, Further results for Perron-Frobenius theorem for nonegative tensors, SIAM J. Matrix Anal. Appl. 31 (2010) 2517-2530. doi:10.1137/090778766

[28] X. Yuan, J. Shao and H. Shan, Ordering of some uniform supertrees with larger spectral radii, Linear Algebra Appl. 495 (2016) 206-222. doi:10.1016/j.laa.2016.01.031

[29] X. Yuan, M. Zhang and M. Lu, Some upper bounds on the eigenvalues of uniform hypergraphs, Linear Algebra Appl. 484 (2015) 540-549. doi:10.1016/j.laa.2015.06.023 
[30] J. Zhang and J. Li, The maximum spectral radius of $k$-uniform hypergraphs with $r$ pendent vertices, Linear Multilinear Algebra 67 (2019) 1062-1073. doi:10.1080/03081087.2018.1442811

[31] J. Zhou, L. Sun, W. Wang and C. Bu, Some spectral properties of uniform hypergraphs, Electron. J. Combin. 21 (2014) \#P4.24.

Received 14 August 2018

Revised 24 April 2019

Accepted 24 April 2019 